\title{
Refractory epilepsy and mitochondrial dysfunction due to GM3 synthase deficiency
}

\author{
Konstantina Fragaki1 ${ }^{1,2,9}$, Samira Ait-El-Mkadem ${ }^{1,2,9}$, Annabelle Chaussenot ${ }^{1}$, Catherine Gire ${ }^{3}$, \\ Raymond Mengual $^{4}$, Laurent Bonesso ${ }^{4}$, Marie Bénéteau ${ }^{5}$, Jean-Ehrland Ricci ${ }^{5}$, Valérie Desquiret-Dumas ${ }^{6,7}$, \\ Vincent Procaccio ${ }^{6,7}$, Agnès Rötig ${ }^{8}$ and Véronique Paquis-Flucklinger ${ }^{\star}, 1,2$
}

We report two children, born from consanguineous parents, who presented with early-onset refractory epilepsy associated with psychomotor delay, failure to thrive, blindness and deafness. Polarographic and spectrophotometric analyses in fibroblasts and liver revealed a respiratory chain (RC) dysfunction. Surprisingly, we identified a homozygous nonsense mutation in the GM3 synthase gene by using exome sequencing. GM3 synthase catalyzes the formation of GM3 ganglioside from lactosylceramide, which is the first step in the synthesis of complex ganglioside species. Mass spectrometry analysis revealed that the complete absence of GM3 ganglioside and its biosynthetic derivatives was associated with an upregulation of the alternative globoside pathway in fibroblasts. The accumulation of Gb3 and Gb4 globosides likely has a role in RC dysfunction and in the decrease of mitochondrial membrane potential leading to apoptosis, which we observed in fibroblasts. We show for the first time that GM3 synthase deficiency, responsible for early-onset epilepsy syndrome, leads to a secondary RC dysfunction. Our study highlights the role of secondary mitochondrial disorders that can interfere with the diagnosis and the evolution of other metabolic diseases.

European Journal of Human Genetics (2013) 21, 528-534; doi:10.1038/ejhg.2012.202; published online 19 September 2012

Keywords: refractory epilepsy; respiratory chain; mitochondrial disease; exome sequencing; GM3 synthase

\section{INTRODUCTION}

Respiratory chain $(\mathrm{RC})$ disorders encompass a wide range of clinical presentations but they affect mainly organs with high-energy requirement such as brain. In a recent series, 66 patients (90\%) among 73 children affected by a mitochondrial disease presented clinical signs of cerebral involvement. ${ }^{1}$ Mitochondrial disorders are also increasingly being recognized as the causes of epilepsy associated with encephalopathy. In the series of 73 children, seizures were observed in 25 patients $(34 \%)^{1}$ and in $61 \%$ of cases in another cohort of 38 children with mitochondrial encephalopathies. ${ }^{2}$ Focal epilepsy seems to be predominant in adults, ${ }^{3}$ whereas pediatric series report a great predominance of generalized epilepsy syndromes.,4,5 Epilepsy of mitochondrial origin is often associated with other symptoms. Among 56 children with RC disorders and epileptic phenotypes, seizures were associated with failure to thrive, psychomotor delay, ataxia or mutisystemic dysfunction in $82.5 \%$ of cases. ${ }^{5}$ Furthermore, $60 \%$ of patients had several seizure types, recurrent status epilepticus and myoclonic epilepsy being clearly overrepresented $(37.5 \%$ and $32 \%$, respectively). ${ }^{5}$ Epilepsy is always difficult to control and the occurrence of intractable seizures is consistently associated with severe neurologic deterioration. ${ }^{2}$ Nevertheless, diagnosis of RC disorders remains challenging for several reasons. First, technical problems can give rise to artifactually low enzyme activities. Second, RC defects frequently have a tissue-specific expression. Last, deficiency of RC complexes may be secondary to other metabolic disorders.

We report two children, who presented early-onset refractory seizures, failure to thrive and psychomotor delay associated with a RC dysfunction. Interestingly, by using exome sequencing we identified a homozygous nonsense mutation in the GM3 synthase gene, which is involved in the synthesis of complex ganglioside species. We show that GM3 synthase deficiency leads to globoside accumulation, secondary RC deficiency, mitochondrial membrane potential perturbation and increase in apoptotic cell death.

\section{MATERIALS AND METHODS}

Patients

The pedigree of the family is shown in Figure 1A. Blood and tissue samples were obtained after parents had given informed consent.

\section{Cell culture}

Primary fibroblast cultures were established using standard procedures in RPMI supplemented with $10 \%$ fetal bovine serum (FBS), $45 \mu \mathrm{g} / \mathrm{ml}$ uridine and $275 \mu \mathrm{g} / \mathrm{ml}$ sodium pyruvate.

${ }^{1}$ Department of Medical Genetics, National Center for Mitochondrial diseases, Nice Teaching Hospital, Nice, France; ${ }^{2}$ RRCAN UMR7284/INSERM U1081/UNS, School of Medicine, Nice Sophia-Antipolis University, Nice, France; ${ }^{3}$ Department of Neuropediatrics, Nord Hospital, Marseille Teaching Hospital, Marseille, France; ${ }^{4}$ Department of Biochemistry, Nice Teaching Hospital, Nice, France; ${ }^{5}$ INSERM U1065, Centre Méditerranéen de Médecine Moléculaire (C3M), team 3 'Contrôle métabolique des morts cellulaires', Nice, France; ' $U$ MR INSERM U1083/CNRS 6214, Angers University, Angers, France; 7 Department of Biochemistry and Genetics, Angers Teaching Hospital, Angers, France; ${ }^{8}$ INSERM U781, Hôpital Necker-Enfants Malades, Université Paris Descartes, Paris, France

*Correspondence: Professor V Paquis-Flucklinger, IRCAN UMR7284/INSERM U1081/UNS, School of Medicine, Nice Sophia-Antipolis University, 28 av de Valombrose, 06107 Nice cedex 2, France. Tel: +33 4933777 86; Fax: +3349337 70 33; E-mail: paquis@hermes.unice.fr

9These authors contributed equally to this work.

Received 21 May 2012; revised 6 August 2012; accepted 10 August 2012; published online 19 September 2012 

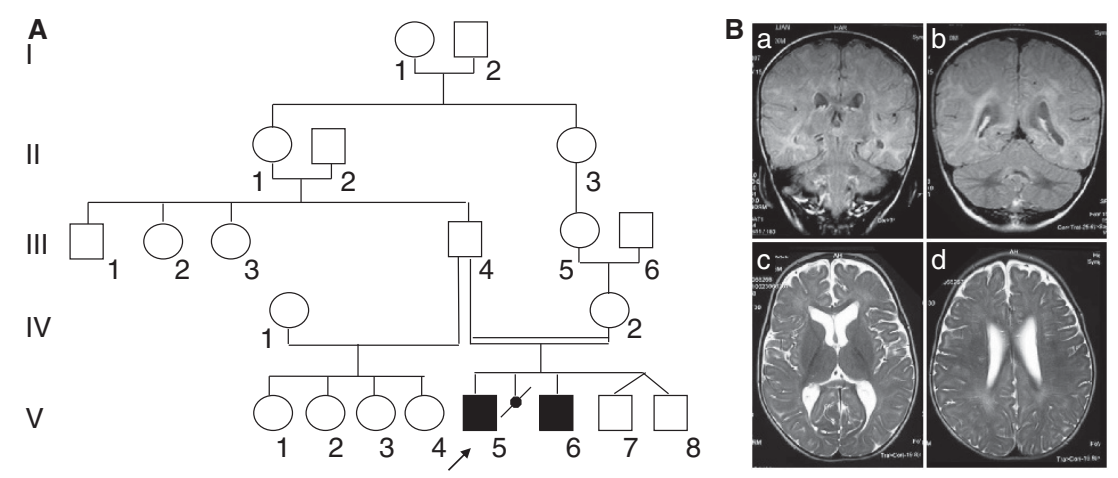

Figure 1 (A) Pedigree of the family. Solid symbols represent clinically affected individuals. (B) Brain MRI data from affected children. (a, b) Patient V-5 at 20 months of age. Coronal fluid-attenuated inversion recovery MRI showed symmetric hyperintensities in the deep and subcortical white matter of the temporal (a) and parietal (b) lobes. (c, d) Patient V-6 at 16 months of age. Axial T2-weighted MRI showed diffuse cortical and calcarine sulcus atrophy (c), diffuse subcortical white matter hyperintensities (c, d) and involvement of posterior periventricular white matter (d).

\section{Polarographic study}

Polarographic studies on fibroblasts of intact cell respiration and digitonin (0.004\%)-permeabilized cells mitochondrial substrate oxidation were carried out as previously described. ${ }^{6}$

\section{OXPHOS spectrophotometric measurements}

Enzymatic spectrophotometric measurements were performed at $37^{\circ} \mathrm{C}$ on muscle and liver crude homogenates and fibroblasts according to the standard procedures. ${ }^{6} \mathrm{CoQ}_{10}$ deficiency was assayed as previously described..$^{7,8}$

\section{Protein measurement}

Proteins were measured according to Bradford microassay. ${ }^{9}$

\section{Molecular analysis}

mtDNA quantification and long-range PCR were performed as described. ${ }^{10,11}$ The search for mtDNA heteroplasmic mutations was done by using Surveyor method. ${ }^{12}$

Microsatellite DNA markers flanking 17 genes involved in the $\mathrm{CoQ}_{10}$ biosynthesis pathway were analyzed. ${ }^{13}$ In the two affected children, we found homozygosity at the PDSS2 (NM_020381) and ADCK4 (NM_001142555) loci. Direct sequencing of these two genes revealed no mutation.

\section{Exome sequencing}

Genomic DNA $(3 \mu \mathrm{g})$ was fragmented by sonication. Exome targets were enriched with the SureSelect Human All Exon Kit, v2, $46 \mathrm{Mb}$ (Agilent technologies, Santa Clara, CA, USA), and sequenced on the Illumina HiSEQ 2000 platform (Illumina, San Diego, CA, USA). Raw image files were processed by the Illumina Real Time Analysis pipeline (version 1.9) for base calling and generating the read sets. The bioinfomatics analysis of sequencing data was based on the Illumina CASAVA pipeline (version 1.7). CASAVA performs alignment of the $2 \times 75 \mathrm{bp}$ paired-end sequence reads to the hg19 reference genome, calls the SNPs based on the allele calls and read depth, and detects variants (SNPs and Indels). The alignment algorithm used was ELAND (version 2). Only the positions included in the bait coordinates were conserved.

For mutation validation, exon 6 of GM3S (SIAT9 or ST3Gal5; NM_003896.3) was sequenced by standard procedures.

\section{Mass spectrometric analysis}

Gangliosides and globosides extraction from the pellet of fibroblasts was proceeded as described ${ }^{14}$ with minor modifications. The aqueous upper layers from two extractions (with mixture of water-methanol-chloroform, $\mathrm{W}: \mathrm{M}: \mathrm{C}=2: 2: 1)$ were collected. Two volumes of water were added to precipitate polyglycoceramides. The dried pellet was resuspended with methanol/water $(\mathrm{M}: \mathrm{W}=1: 1)$ and analyzed in reflector-positive and -negative modes on MALDI-TOF/TOF mass spectrometer Ultraflex III (Bruker Daltonics, Bremen, Germany) from 700 to $3000 \mathrm{Da}$ (Dalton). The sample was spotted and mixed with dihydroxide benzoic acid matrix on steel target plate.

Measurement of mitochondrial membrane potential in fibroblasts Cells were resuspended in PBS, FBS 5\%,50 mM KCl and incubated with $20 \mathrm{~nm}$ cationic fluorescent dye DiOC6(3) for $30 \mathrm{~min}$. In some wells, oligomycin (8 $\mu \mathrm{g} /$ $\mathrm{ml})$ or mClCCP $(10 \mu \mathrm{M})$ was added before incubation, either to inhibit ATP synthesis or to dissipate the proton gradient. After washing, cells were set in tubes containing propidium iodide $(8 \mu \mathrm{g} / \mathrm{ml})$ to check membrane integrity. The fluorescent intensity was read using a flow cytometer (Becton Dickinson, Le-Pont-De-Claix, France). Laser excitation of DiOC6(3) was at $484 \mathrm{~nm}$ and emission was read at $511 \mathrm{~nm}$. Propidium iodide fluorescence was detected at $610 \mathrm{~nm}$. Fluorescence analysis was performed using Cell Quest software (Becton Dickinson).

\section{Annexin $\mathrm{V}$ assay}

Fibroblasts were treated with $1 \mu \mathrm{M}$ rotenone (Sigma-Aldrich, St Louis, MO, USA) dissolved in ethanol/DMSO (50:50, v/v). Twenty-four hours later, cell pellets were resuspended in $200 \mu \mathrm{l}$ of buffer $(150 \mathrm{~mm} \mathrm{NaCl}, 10 \mathrm{~mm}$ Hepes pH7.4, $5 \mathrm{~mm} \mathrm{KCl}, 1 \mathrm{~mm} \mathrm{MgCl}, 1.8 \mathrm{~mm} \mathrm{CaCl}_{2}$ ) and incubated with recombinant Annexin V-phycoerythrin (BD Pharmingen, Le-Pont-De-Claix, France) for $10 \mathrm{~min}$ at room temperature. A volume of $0.5 \mu \mathrm{g} / \mathrm{ml}$ of DAPI (Invitrogen, Saint Aubin, France) was added, and samples were analyzed immediately by flow cytometry using a MACSQuant Analyzer (Miltenyi Biotec, Bergisch Gladbach, Germany). Percentage of apoptotic cells corresponds to Annexin V-simple-positive cells and Annexin V/DAPI-double-positive cells.

\section{RESULTS}

Early-onset epilepsy syndrome evocative of a mitochondrial disorder

The index case (V-5) was born at term, eutrophic, after an uncomplicated pregnancy, from healthy consanguineous French parents (Figure 1A). He presented tonic seizures when he was 2month-old, then myoclonus appeared 2 months later. His epilepsy became severe and pharmacoresistant with polymorphic seizures and nonconvulsive status epilepticus. Psychomotor development was delayed. He has acquired head control at 15 months of age, and was unable to sit unsupported, but he had good eye contact and he smiled interactively. Poor feeding, recurrent vomiting and failure to thrive were rapidly observed and were slightly improved by gastrostomy. Choreoathetoid movements began at 14 months of age. He presented a neurological deterioration at 3 years and a half. At the age 
of 6 , the patient presented hypotonic tetraparesis, permanent choreoathetosis, nonconvulsive status epilepticus and visual impairment. Weight and height were below the third percentile $(10.3 \mathrm{~kg}$ and $93.5 \mathrm{~cm}$, respectively). Laboratory investigations revealed an increase in plasma lactate concentrations $(2.6 \mathrm{~mm} / 1,0.5<$ normal $<2.0)$. Metabolic screening, including the search for lysosomal storage diseases, showed no other abnormalities. Symmetrical white matter hyperintensities and diffuse cortical atrophy were observed on brain magnetic resonance imaging (MRI) (Figures $1 \mathrm{Ba}$ and $\mathrm{b}$ ). The second child (V-6) presented the same clinical presentation. Epilepsy started at 9 months including severe, pharmacoresistant and polymorphic seizures including myoclonic episodes. He presented a developmental delay with a mild axial hypotonia, a head control completely acquired at 11 months and sitting at 15 months. Because of feeding difficulties, a gastrostomy was placed at the age of 18 months with little improvement. At 15 months, he presented choreoathetoid movements. The neurologic status further deteriorated at 26 months. At 3 years of age, ophtalmologic examination showed a mild bilateral optic nerve pallor. At the age of 4 years, weight and height were below the third percentile $(8.5 \mathrm{~kg}$ and $82 \mathrm{~cm}$, respectively). The patient was hypotonic, blind and deaf. He presented permanent abnormal involuntary movements, and frequent nonconvulsive status epilepticus episodes. Brain MRI also showed symmetrical white matter involvement and cortical atrophy, mainly in occipital visual cortex (Figures $1 \mathrm{Bc}$ and $\mathrm{d}$ ).

\section{$\mathrm{RC}$ dysfunction in fibroblasts and liver}

The clinical presentation associated to lactate elevation and absence of other metabolic abnormalities led us to search for RC deficiency. In fibroblasts of patient V-5, we observed a decrease of both oxygen consumption and glutamate/malate oxidation (Table 1A). Nevertheless, spectrophotometric analysis did not reveal any deficiency of the RC complexes (Table 1B). In liver, the activities of CI + III and $\mathrm{CII}+\mathrm{III}$ were reduced in absolute values (Table $1 \mathrm{C}$ ). This result pointed to a deficiency in quinone-dependent complexes $(\mathrm{CI}+\mathrm{III}$ and CII + III) in patient's liver. This hypothesis was supported by the restoration of complex II + III activity after incubation with exogenous ubiquinone (decylubiquinone) (Supplementary Figure 1). ${ }^{7,8}$ Unfortunately, we did not have enough material to perform a direct measurement of $\mathrm{CoQ}_{10}$ content in the patient's liver. We analyzed microsatellite DNA markers flanking 17 genes known to be involved in $\mathrm{CoQ}_{10}$ biosynthesis pathway and found homozygosity for the two affected children at the PDSS2 and ADCK4 loci. Nevertheless, direct sequencing of these two genes revealed no mutation. The histological analysis of muscle showed lipid accumulation and the enzymatic spectrophotometric measurements revealed no RC deficiency (data not shown). The parents refused the tissue biopsies from the second affected child (V-6).

We found no mtDNA depletion in muscle or in liver from patient V-5 (44\% and $78 \%$ of age-matched controls, respectively). We also excluded mtDNA deletion(s) and mtDNA heteroplasmic mutations. ${ }^{11,12}$

\section{Identification of a homozygous loss-of-function mutation in the GM3 synthase gene by exome sequencing}

To identify the causative nuclear gene mutation, we sequenced the exome of patient $\mathrm{V}-5$. The procedure yielded $7.66 \mathrm{~Gb}$ of mappable sequence, with a mean depth coverage of $73 \times$ and $75.6 \%$ of bases covered to at least $25 \times$. From the 39364 identified SNPs, a serial stringent (loss-of-function) filtering led us to identify a single homozygous nonsense mutation in exon 6 of the GM3 synthase gene (c.862C > T; p.Arg288*). Surprisingly, the protein product catalyzes the formation of GM3 ganglioside from lactosylceramide, which is the first step in the synthesis of complex ganglioside species (Figure 2a). The mutation cosegregated with the disease and was homozygous in the two affected individuals and heterozygous in parents and non affected twins (Figure 2b). The same mutation has been described previously in an Amish family, including several individuals

Table 1 Respiratory chain analysis in fibroblasts and liver from patient V-5

Oxygen consumption

\begin{tabular}{|c|c|c|c|c|}
\hline \multirow[b]{3}{*}{ (A) Oxygraphic analysis on fibroblasts } & & & & \\
\hline & \multirow[b]{2}{*}{ Intact cells } & \multicolumn{3}{|c|}{ Digitonine permeabilized cells } \\
\hline & & Glutamate + malate & Succinate & G3P \\
\hline Control values ( $\mathrm{nmol} \mathrm{O}_{2} / \mathrm{min} / \mathrm{mg}$ of proteins) & $5.90-13.80$ & $8.00-16.60$ & $8.00-15.80$ & $4.90-13.50$ \\
\hline Patient V-5 & 5.59 & 6.73 & 10.14 & 5.24 \\
\hline
\end{tabular}

(B) Spectrophotometric analysis on fibroblasts

\begin{tabular}{|c|c|c|c|c|c|c|c|c|}
\hline Enzymatic activities & I & II & III & IV & $V$ & $I I+I I I$ & $G 3 P+I I I$ & $C S$ \\
\hline Control values (nmol/min/mg of proteins) & $9.0-27.1$ & $18.5-33.4$ & $57.4-176.2$ & 109.9-252.7 & $22.0-46.2$ & $13.3-37.2$ & $5.6-23.0$ & $74.7-161.1$ \\
\hline Patient V-5 & 17.2 & 24.0 & 86.7 & 229.2 & 43.5 & 17.6 & 7.7 & 144.2 \\
\hline
\end{tabular}

(C) Spectrophotometric analysis on the liver

\begin{tabular}{lcccccccc} 
Enzymatic activities & $I$ & $I I$ & III & I & III III \\
\hline Control values (nmol/min/mg of proteins) & $13-41$ & $78-267$ & $124-246$ & $146-316$ & $41-117$ & $33-73$ & $39-113$ & $43-107$ \\
Patient V-5 & 32.9 & 161.4 & 152.0 & 183.1 & 84.5 & 19.9 & 25.8 & 131.3
\end{tabular}

(A) Polarographic analysis of the respiratory chain in fibroblasts. Results are expressed as extreme absolute values or absolute values for controls or patients, respectively. Values are expressed in nanomoles of oxygen per minute per milligram of proteins.

(B and C) Spectrophotometric analysis of the respiratory chain enzyme activities in (B) fibroblasts and in (C) liver. Results are expressed as extreme absolute values or absolute values for controls or patients, respectively. Values are expressed in nanomols of substrate per minute per milligram of proteins (lowered values are in grey). 
a

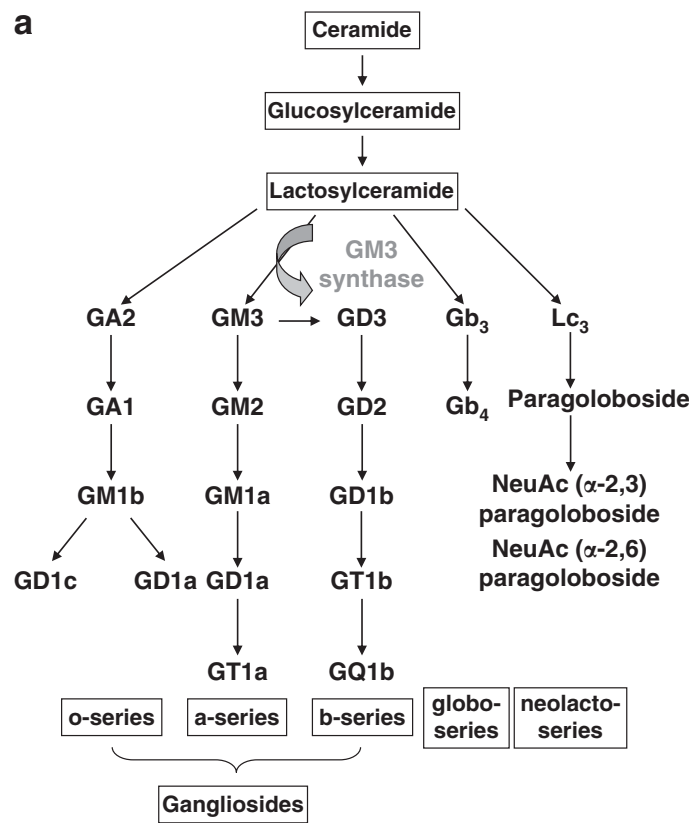

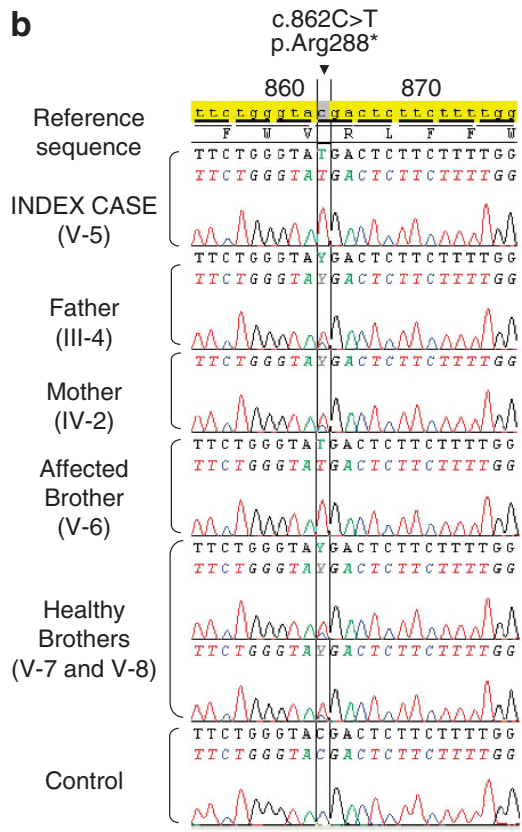

Figure 2 (a) Schematic view of ganglioside biosynthesis. (b) Identification of a homozygous nonsense mutation in the GM3 synthase gene. Sequence analysis of the GM3 synthase gene showing the segregation of the c.862C > T (p.Arg288*) mutation in the family.

presenting an infantile-onset epilepsy syndrome. ${ }^{15}$ This study is the only one found in the literature that reports a mutation in GM3 synthase but without any reference to a mitochondrial dysfunction.

A less stringent filtering identified 15 other homozygous variations (10 missense, 1 splice-site disruption and 4 indels). None of the corresponding genes encoded a predicted mitochondrial protein after an analysis by MitoProt, TargetP and MitoCarta, and none had a function suggesting that the variants identified may contribute to the mitochondrial phenotype observed.

\begin{abstract}
Absence of GM3 and increase of Gb3 and Gb4 species in patient's fibroblasts by mass spectrometric analysis

To confirm the role of GM3 synthase deficiency, we determined the biochemical effect of the mutation in the fibroblasts of patient V-5 by using mass spectrometry (MS) analysis. A representative profile from normal fibroblasts showing the presence of lactosylceramide and gangliosides (GM3, GA2) in reflector-positive mode is shown in Figure $3 \mathrm{a}$. In patient V-5, the ganglioside pathway was not detectable while the globoside pathway was upregulated (Figure 3b). These results were confirmed in reflector-negative mode analysis, which optimizes sialylated derivated. The presence of peaks corresponding to gangliosides (GM3, GM2, GM1 and GD3) was observed in control fibroblasts but these peaks were undetectable or weak in patient V-5, suggesting that these compounds were absent or weakly present (data not shown).
\end{abstract}

Decrease of mitochondrial membrane potential and increase of apoptosis in patient's fibroblasts

As alteration of glycosphingolipid (GSL) flux can affect membrane composition, we examined the effects of GM3 synthase deficiency on mitochondrial membrane potential $(\Delta \Psi \mathrm{m})$. Flow cytometry experiments with DiOC6(3) fluorescent dye showed a significant decrease of mitochondrial membrane potential in fibroblasts of patient $\mathrm{V}-5$ compared with control cells both in basal conditions and after oligomycin incubation (stade IV, non phosphorylating conditions) (Figure 4a).

Gangliosides are known to trigger apoptosis. Flow cytometry experiments showed increased apoptosis in patient's fibroblasts compared with control cells. Patient fibroblasts were significantly more sensitive to rotenone treatment that induced a 4.5 -fold increase in Annexin $\mathrm{V}+/ \mathrm{DAPI}$ - cell population whereas the increase was only 1.8 -fold in control fibroblasts $(P<0.04)$ (Figure $4 \mathrm{~b}$ ). Other evidence for sensitivity of patient fibroblasts to cell death comes from the slight but significant $\sim 2$-fold increase in percentage of AnnexinV $+/$ DAPI + cells observed at the basal state compared with control cells $(5.7 \pm 1.4 \%$ vs $2.8 \pm 0.3 \%$ respectively, $P<0.03$ ).

\section{DISCUSSION}

We report two children presenting pharmacoresistant epilepsy associated with psychomotor delay and failure to thrive. Clinical symptoms associated to lactate elevation and absence of other metabolic abnormalities led us to search for RC deficiency. In fibroblasts, we observed a decrease of both oxygen consumption and glutamate/malate oxidation, possibly ascribed to an impairment in electron movement through the RC as previously described. ${ }^{16}$ In liver, we observed that activities of $\mathrm{CI}+\mathrm{CIII}$ and $\mathrm{CII}+\mathrm{CIII}$ were decreased. Coenzyme Q (CoQ) shuttles electrons from complex I and complex II to complex III. ${ }^{13}$ The hypothesis of a deficiency in quinone-dependent complexes was strengthened by the restoration of complex II + III activity after incubation with exogenous ubiquinone, but we found no mutation in genes known to be involved in the $\mathrm{CoQ}_{10}$ biosynthesis pathway. At this stage, we were unable to determine whether this deficiency in $\mathrm{CI}+\mathrm{CIII}$ and $\mathrm{CII}+\mathrm{CIII}$ was related to a primary defect in the biosynthesis pathway of $\mathrm{CoQ}_{10}$ or a secondary problem to another etiology. As several authors have reported a positive effect of $\mathrm{CoQ}_{10}$ supplementation, the two affected children were treated with ubiquinone $(2000 \mathrm{mg} /$ day $)$. Subjective improvement was observed after 2 months of treatment and consisted of a better eye contact and increased interactivity with a 

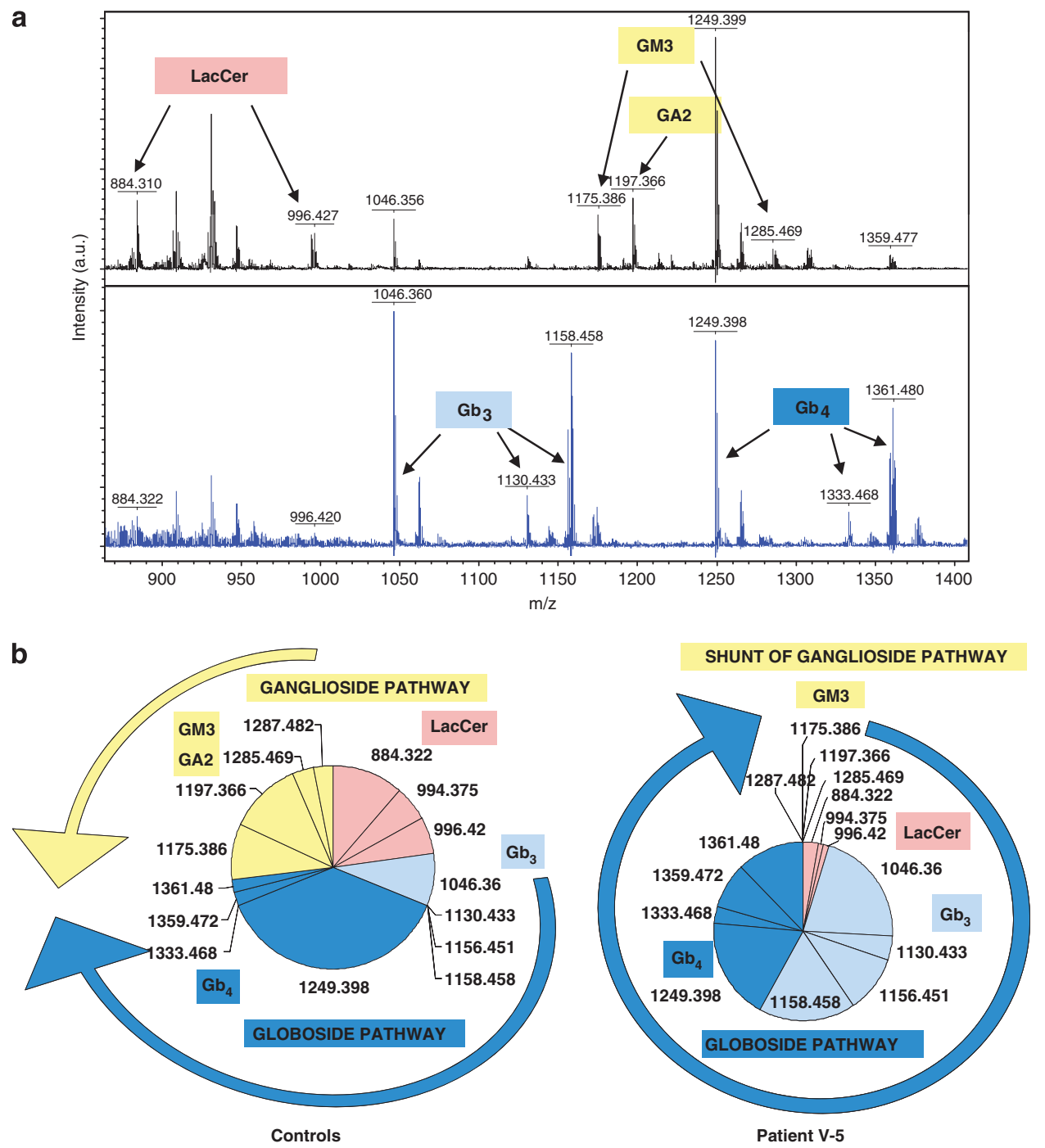

Figure $3 \mathrm{MS}$ analysis in the fibroblasts of patient V-5. (a) MALDI-TOF spectrometry analysis in positive mode. Representative spectra from control (upper panel), maximal unit $10^{4}$ and, patient (lower panel), maximal unit $0.5 \times 10^{4}$. (b) Schematic lactoceramide pathway in control (left) and patient (right).
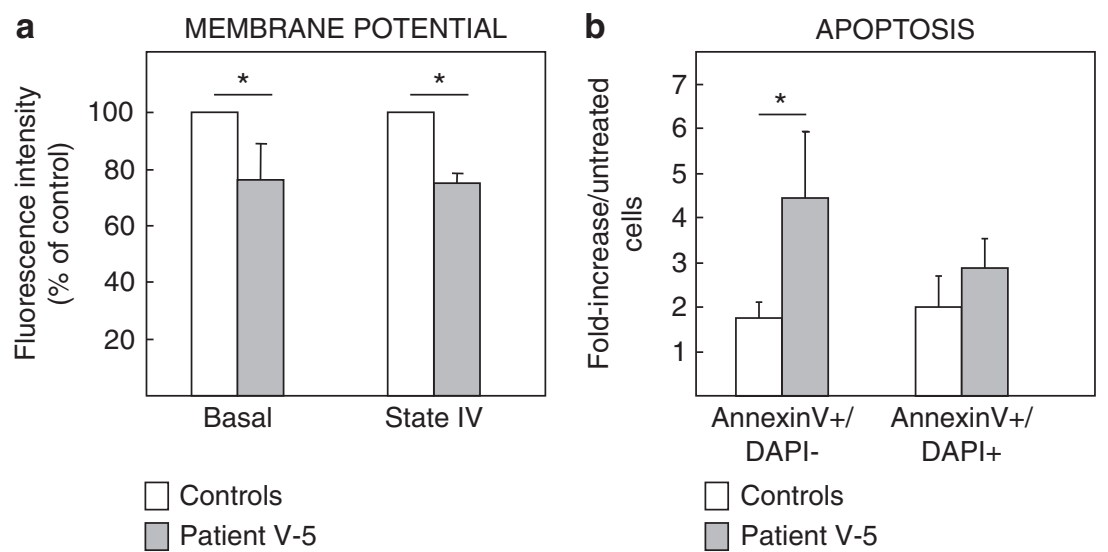

Figure 4 (a) Measurement of mitochondrial membrane potential in fibroblasts of patient $V-5$. Mitochondrial membrane potential was estimated by flow cytometry analysis of the catonic dye DiOC6(3) on control and patient fibroblasts. Fluorescence was measured on phosphorylating (basal) and non phosphorylating (state IV, not coupled to ATP synthesis) conditions. (b) Analysis of apoptosis in fibroblasts of patient V-5. Apoptosis was induced by $24 \mathrm{~h}$ rotenone treatment. Ratios of apoptosis were established using untreated cell extracts has having a value of 1 . Columns are the mean from three independently performed experiments; bars, $\mathrm{SE} ;{ }^{*} P<0.05$ versus control fibroblasts. 
slight decrease in the number of seizure episodes. Nevertheless, after a few months, symptoms worsened with a return to the initial clinical condition.

To identify the causative gene mutation, we performed exome sequencing. Surprisingly, we identified a homozygous loss-of-function mutation in the GM3 synthase gene which is involved in the synthesis of complex ganglioside species. The same mutation was identified in a large Old Order Amish pedigree, despite a different annotation based on a previous reference sequence, and was responsible for a similar clinical presentation with early-onset pharmacoresistant epilepsy, recurrent vomiting and failure to thrive. ${ }^{15}$ Patients also presented abnormal movements with choreoathetoid component. Developmental stagnation was profound with a regression episode. There was some evidence of optic atrophy to explain blindness while retinal function appeared to be normal. ${ }^{17}$ Farukhi et al also suggested a cortical visual impairment. ${ }^{17}$ Our study strengthens this hypothesis. First, the ophthalmologic examination of the youngest affected child showed a bilateral pallor of the optic nerve which is too mild compared with the severity of blindness. Second, brain MRI showed occipital white matter abnormalities and significant atrophy of occipital cortex, especially calcarine sulcus, where primary visual cortex is located. Otherwise, signal abnormalities were also observed in the deep and subcortical white matter of temporal and parietal lobes, corresponding to a leukodystrophy. In the Amish pedigree, only diffuse atrophy was found on the brain MRI. ${ }^{15}$

Gangliosides are sialic acid-containing GSLs. ${ }^{18}$ GM3 synthase catalyzes the formation of GM3 from lactosylceramide, corresponding to the first step of complex ganglioside synthesis (a- and b-series). Alternative enzymatic modification of lactosylceramide leads to the synthesis of alternative derivatives (o-, globo- and neolacto-series). Although the relationship between defects in ganglioside catabolism and a range of lysosomal storage diseases is well documented, only three human diseases are associated with mutations in enzymes involved in de novo GSL synthesis and patients are extremely rare. ${ }^{15,19,20}$ The identification of the p.Arg288* mutation in a Amish pedigree is the only example of a GM3 synthase deficiency reported in the literature but without any reference to a mitochondrial dysfunction. ${ }^{15}$ Contrary to our family, no reference to deafness was reported by Simpson et al. ${ }^{15}$ Nevertheless, mice lacking GM3 synthase exhibit complete hearing loss due to selective degeneration of the organ of Corti demonstrating that GM3 is essential for acquisition and maintenance of hearing. ${ }^{21}$ In the Amish family, the affected children completely lacked GM3 and its downstream biosynthetic derivatives in plasma. Levels of lactosylceramide, the immediate precursor of GM3, and alternative derivatives of GM3 (globo- and neolacto-series) were increased. Interestingly, in the fibroblasts of patient V-5, the complete absence of gangliosides (GM3 and GA2) was not associated with an increase in lactosylceramide. Only evidence of increased flux through the globoside pathway (Gb3 and Gb4) was observed.

In vitro studies on isolated mitochondria showed that ceramide exposure inhibits CIII of the RC, resulting in reactive oxygen species production, release of cytochrome $c$ and caspase activation. ${ }^{22,23}$ The direct effect of ceramide on mitochondria was reproduced with GD3 ganglioside that leads to a marked loss of mitochondrial $\Delta \Psi \mathrm{m}$ and apoptosis. ${ }^{24}$ These studies led us to assume that the mitochondrial dysfunction that we observed was not an artefact but was secondary to the GM3 synthase deficiency. This hypothesis was strengthened by the demonstration of a significant decrease in mitochondrial membrane potential and a significant increase in apoptosis, in response to rotenone, in the patient's fibroblasts compared with control cells. These results could be a direct consequence of RC deficiency, leading to the impairment of normal mitochondrial electron flow and proton pumping, including a drop in $\Delta \Psi \mathrm{m}$ as previously described. ${ }^{16,25}$ Cortan et $a l^{25}$ reported that MELAS fibroblasts show a significant reduction of $\Delta \Psi \mathrm{m}$ associated with secondary $\mathrm{CoQ}_{10}$ deficiency that triggers mitochondria degradation by mitophagy. To date, there is no published study that has tested the effect of globosides on mitochondria, but it is likely that the accumulation of $\mathrm{Gb} 3$ and $\mathrm{Gb} 4$ found in fibroblasts of the patient has a role in the mitochondrial dysfunction that we observed.

In conclusion, we demonstrated that GM3 synthase deficiency, responsible for early-onset symptomatic epilepsy syndrome, leads to a secondary mitochondrial dysfunction. It is very important to diagnose secondary RC deficiencies because energetic defect can influence the evolution and the prognosis of metabolic diseases. We have recently reported that quinone deficiency secondary to propionic acidemia can be responsible for fatal cardiac complications. ${ }^{26}$ Last, only the identification of the causative gene allows proposing an appropriate genetic counseling and a reliable prenatal diagnosis.

\section{CONFLICT OF INTEREST}

The authors declare no conflict of interest.

\section{ACKNOWLEDGEMENTS}

We thank Charlotte Cochaud for technical help and Sylvie Bannwarth for helpful discussion.

1 Debray F, Lambert M, Chevalier I et al: Long-term outcome and clinical spectrum of 73 pediatric patients with mitochondrial diseases. Pediatrics 2007; 119: 722-733.

2 Khurana D, Salganicoff L, Melvin J et al: Epilepsy and respiratory chain defects in children with mitochondrial encephalopathy. Neuropediatrics 2008; 39: 8-13.

3 Canafoglia L, Francheschetti S, Antozzi C et al: Epileptic phenotypes associated with mitochondrial disorders. Neurology 2001; 56: 1340-1346.

4 Lee Y, Kang H, Lee J et al: Mitochondrial respiratory chain defects: underlying etiology in various epileptic conditions. Epilepsia 2008; 44: 701-707.

5 El Sabbagh S, Lebre A, Bahi-Buisson N et al: Epileptic phenotypes in children with respiratory chain disorders. Epilepsia 2010; 51: 1225-1235.

6 Rustin P, Chretien D, Bourgeron T, Gerard B, Rotig A, Saudubray J: Biochemical and molecular investigations in respiratory chain deficiencies. Clin Chem Acta 1994; 228: 31-51.

7 Lerman-Sagie T, Rustin P, Lev D et al: Dramatic improvement in mitochondrial cardiomyopathy following treatment with idebenone. J Inherit Metab Dis 2001; 24: 28-34.

8 Leshinsky-Silver E, Levine A, Nissenkorn A et al: Neonatal liver failure and Leigh syndrome possibly due to the CoQ-responsive OXPHOS deficiency. Mol Genet Metab 2003; 79: 288-293.

9 Bradford M: A rapid and sensitive method for the quantitation of micrograms quantities of protein utilizing the principle of protein-dye binding. Annal Biochem 1976; 251: 69-72.

10 Rouzier C, Le Guedard-Méreuze S, Fragaki K et al: The severity of phenotype linked to SUCLG1 mutations could be correlated with residual amount of SUCLG1 protein. J Med Genet 2010; 47: 670-676.

11 Paul R, Santucci S, Saunières A, Desnuelle C, Paquis-Flucklinger V: Rapid mapping of mitochondrial DNA deletiond by large fragment PCR. Trends Genet 1996; 12: 131-132.

12 Bannwarth S, Procaccio V, Paquis-Flucklinger V: Surveyor nuclease: a new strategy for a rapid identification of heteroplasmic mitochondrial DNA mutations in patients with respiratory chain defects. Hum Mutat 2005; 25: 575-582.

13 Rotig A, Mollet J, Rio M, Munnich A: Infantile and prediatric quinone deficiency diseases. Mitochondrion 2007; 7S: S112-S121.

14 Lee H, Ann H, Lerno LJ, German J, Lebrilla C: Rapid profiling of bovine and human milk gangliosides by matrix-assisted laser desorption/ionization Fourier transform ion cyclotron resonance mass spectrometry. Int J Mass Spectrom 2011; 305: 138-150.

15 Simpson M, Cross H, Proukakis C et al: Infantile-onset symptomatic epilepsy syndrome caused by a homozygous loss-of-function mutation of GM3 synthase. Nat Genet 2004; 11: 1225-1229.

16 James A, Wei Y-H, Pang C-Y, Murphy M: Altered mitochondrial function in fibroblasts containing MELAS or MERRF mitochondrial DNA mutations. Biochem J 1996; 318: 401-407.

17 Farukhi F, Dakkouri C, Wang H, Wiztnitzer M, Traboulsi E: Etiology of vision loss in ganglioside GM3 synthase deficiency. Ophtalmic Genet 2006; 27: 88-91. 
18 Xu Y-H, Barnes S, Sun Y, Grabowski G: Multi-system disorders of glycosphingolipid and ganglioside metabolism. J Lipid Res 2010; 51: 1643-1675.

19 Fishman P, Max S, Tallman J, Brady R, Maclaren N, Cornblath M: Deficient ganglioside biosynthesis: a novel human sphingolipidosis. Science 1975; 187: 68-70.

20 Dawkins J, Hulme D, Brahmbliatt S, Auer-Grumbach M, Nicholson G: Mutations in SPTLC1, encoding serine palmitoyltransferase, long chain base subunit-1, cause hereditary sensory neuropathy type 1 . Nat Genet 2001; 27: 309-312.

21 Yamashita T, Hashiramoto A, Haluzik M et al: Enhanced insulin sensitivity in mice lacking ganglioside GM3. Proc Natl Acad Sci 2003; 100: 3445-3449.

22 Gudz T, Tserng K, Hoppel C: Direct inhibition of mitochondrial respiratory chain complex III by cell-permeable ceramide. J Biol Chem 1997; 272: 24154-24158
23 Garcia-Ruiz C, Colell A, Mari M, Morales A, Fernadez-Checa J: Direct ineraction of GD3 ganglioside with mitochondria generates reactive oxygen species followed by mitochondrial permeability transition, cytochome $c$ relaese, and caspase activation. FASEB 2000; 14: 847-858.

24 Higuchi Y, Miura T, Kajimoto T, Ohta Y: Effects of disialoganglioside GD3 on the mitochondrial membrane potential. FEBS Lett 2005; 579: 3009-3013.

25 Cortan D, Cordero M, Garrido-Marraver J et al: Secondary coenzyme Q10 deficiency triggers mitochondria degradation by mitophagy in MELAS fibroblasts. FASEB 2011; 25: 2669-2687.

26 Fragaki K, Cano A, Benoist J et al: Fatal heart failure associated with CoQ10 and multiple OXPHOS deficiency in a child wiith propionic acidemia. Mitochondrion 2011; 11: 533-536.

Supplementary Information accompanies the paper on European Journal of Human Genetics website (http://www.nature.com/ejhg) 